\title{
Guanosine Triphosphate
}

National Cancer Institute

\section{Source}

National Cancer Institute. Guanosine Triphosphate. NCI Thesaurus. Code C536.

A purine nucleotide triphosphate comprised of a guanine and a triphosphate moiety bound to a ribose moiety at its 1 ' and 5' sites, respectively. Guanosine triphosphate (GTP) can be utilized as a substrate for nucleic acid (DNA and RNA) synthesis, as an energy source for protein synthesis and gluconeogenesis, and as a signaling molecule. 